\title{
Cost-Effectiveness of IL28B Genotype-Guided Protease Inhibitor Triple Therapy versus Standard of Care Treatment in Patients with Hepatitis C Genotypes 2 or 3 Infection
}

\author{
Jonathan A. Bock ${ }^{a}$ Kimberly J. Fairley ${ }^{b}$ Robert E. Smith ${ }^{b}$ Daniel D. Maeng ${ }^{c}$ \\ James M. Pitcavage ${ }^{c}$ Nicholas A. Inverso $^{b}$ Marc S. Williams ${ }^{d}$ \\ ${ }^{a}$ Weis Center for Research, ${ }^{b}$ Department of Gastroenterology, ${ }^{c}$ Center for Health Research, and \\ ${ }^{\mathrm{d}}$ Genomic Medicine Institute, Geisinger Medical Center, Danville, Pa., USA
}

\section{Key Words}

Cost-effectiveness - Decision tree · Hepatitis C virus · IL28B .

Markov modeling $\cdot$ Telaprevir

\begin{abstract}
Background/Aims: Triple therapy [adding protease inhibitors to standard of care (SOC)] dramatically increases treatment response in selected patients with hepatitis $C$ virus (HCV). Interleukin 28B (IL28B) genotyping helps predict responsiveness in these patients; however, the economic implications of IL28B genotyping in HCV genotype 2 or 3 infected patients are unknown. Short- and long-term costs and outcomes of SOC therapy were calculated and used to determine the cost-effectiveness thresholds for using triple therapy in HCV genotype 2 or 3 infected patients. Methods: Costs and outcomes were calculated by conducting cohort simulations on decision trees modeling SOC and triple therapy. Quality-adjusted life expectancies and long-term costs were predicted through Markov modeling. Results: For triple therapy to be cost-effective, sustained virologic response (SVR) rates must improve (depending on age) by 7.91-11.11 and $9.06-12.8 \%$ for HCV genotype 2 and 3 cohorts, respectively. When triple therapy is guided by 2 IL28B variants, a 2.63-3.72\% improvement in SVR is needed for cost-effec-
\end{abstract}

tiveness, and when guided by only one variant, a 1.4-8.91\% improvement is needed. Conclusions: Markov modeling revealed that modest increases in SVR rates from IL28B-guided triple therapy can lead to both lower costs and better health outcomes than SOC therapy in the long run.

(c) 2014 S. Karger AG, Basel

\section{Introduction}

Chronic hepatitis $\mathrm{C}$ virus (HCV) infection can burden individuals with devastating complications that include sequelae of decompensated cirrhosis, hepatocellular carcinoma (HCC), and extra-hepatic manifestations. In the US alone, the CDC estimates 2.7-3.9 million persons (prevalence rate $1.0-1.5 \%$ of the population) are infected [1]. The incidence of infection in 2010 was estimated to be 17,000 persons [1]. Hepatitis C remains the leading cause of cirrhosis and hepatocellular cancer in Western countries and creates a significant healthcare-related economic burden [2].

Until recently, the standard of care (SOC) treatment for all genotypes of $\mathrm{HCV}$ infection was pegylated interferon combined with ribavirin, with therapy durations varying from 24-48 weeks or even longer. SOC for most

\section{KARGER}

E-Mail karger@karger.com

www.karger.com/phg
(C) 2014 S. Karger AG, Basel

$1662-4246 / 14 / 0176-0306 \$ 39.50 / 0$
Marc S. Williams

Genomic Medicine Institute, Geisinger Medical Center

100 N. Academy Avenue

Danville, PA 17822 (USA)

E-Mail mswilliams1@geisinger.edu 
HCV genotypes has remained unaltered; however, since 2011, the SOC for patients infected with HCV genotype 1 has been triple therapy, which combines $24-48$ weeks of pegylated interferon and ribavirin with up to 12 weeks of a protease inhibitor such as telaprevir or boceprevir.

Rates for sustained virologic response (SVR), which is the optimal outcome of therapy, in treatment-naïve patients with HCV genotype 1 infection have reached 40 $50 \%$ under dual therapy with pegylated interferon and ribavirin, yet recent studies show that the addition of telaprevir or boceprevir increases SVR rates to $70-80 \%$ in such patients [3]. HCV genotypes 2 and 3 have been shown to be more responsive to SOC therapy compared to genotype 1. Overall SVR rates to SOC treatment for HCV genotypes 2 and 3 infected patients have been documented to reach $80 \%$ [4]. Yet, in infected individuals that do not attain undetectable HCV RNA levels after 4 weeks of treatment [otherwise known as rapid virologic response (RVR)], SVR rates only approach 62 and $46 \%$, in HCV genotypes 2 and 3, respectively [5]. This suggests an opportunity for targeted triple therapy to improve SVR rates for selected patients with HCV genotype 2 or 3 infection.

One target to select patients for more intensive therapy can be the host interleukin 28B (IL28B) genotype. Previous studies have demonstrated that alleles at 2 SNPs, rs12979860 and rs8099917, are associated with the therapeutic response in HCV genotypes 1-3 infected individuals [5, 6]. Moreover, rs12979860 appears to be the dominant SNP predicting responsiveness to interferon therapy, with the CC genotype identifying relative responsiveness (i.e. an increased frequency of sustaining viral clearance at 6 months post treatment) and the TT genotype indicating relative resistance (i.e. a decreased frequency of sustaining viral clearance at 6 months post treatment). When the heterozygous state (CT) exists at SNP rs12979860 (intermediate response phenotype), a second SNP, rs8099917, has a significant role in determining responsiveness to interferon-based therapy in these patients. In this circumstance, homozygous TT at rs 8099917 predicts better response to therapy than either homozygous GG or heterozygous TG at SNP rs8099917 [6]. This dichotomous status has been demonstrated in HCV genotype 2/3-treated patients. Of those obtaining a SVR with SOC therapy (overall 78\%), 87.4\% were homozygous CC at position rs12979860 as compared to $73.1 \%$ homozygous TT at the same position [7].

Significant improvements in response rates to therapy have been realized with the adjunctive use of protease inhibitors for HCV genotype 1 infected patients $[8,9]$.
Chayama et al. [10] was among the earliest investigators to evaluate triple therapy in HCV genotype 1 infected patients with variations in IL28B SNPs. Homozygosity (TT) at the second SNP, rs8099917, predicted a favorable response to therapy with an SVR rate of $94 \%$.

In vitro data suggests that telaprevir is also active against HCV genotypes 2 and 3 [11]. A phase II clinical trial was conducted to assess telaprevir's ability to increase in vivo SVR rates. Triple therapy with 15 days of telaprevir and 24 weeks of pegylated interferon/ribavirin resulted in SVR rates of $100 \%$ compared to $67 \%$ in patients with genotype 2 and 3 treated with dual therapy. A second population in this study received 15 days of telaprevir monotherapy, which produced a 3.27 and 0.54 $\log 10 \mathrm{IU} / \mathrm{ml}$ decrease in viral load for genotype 2 and 3 patients, respectively [12]. Although the study used nonstandard treatment protocol (15 days of telaprevir therapy), SVR rates in genotype 2 infected patients were $100 \%$ compared to $89 \%$ in the telaprevir and pegylated interferon/ribavirin versus pegylated interferon/ribavirin alone treatment arms, respectively. In genotype 3 infected patients, the SVR rate was $23 \%$ higher in the triple therapy group as well [12]. Therefore, it is reasonable to assess the economic implications of triple therapy in both genotype 2 and 3 infected patients.

In our transplant center experience, telaprevir has been used off-label for the treatment of HCV genotype 2 infected patients who previously failed dual therapy and have demonstrated unfavorable host genetics based on IL28B genotyping. An SVR has been achieved in many patients with the addition of triple therapy. No studies have evaluated the usefulness of IL28B genotyping in response to triple therapy for HCV genotype 2 or 3 infection. Therefore, it is reasonable to consider protease inhibitor therapy in patients anticipated to fail dual therapy and to evaluate the economic implications of such treatment.

Triple therapy can be a significant financial burden to the patient and the health system. A recent study noted that, depending on the duration of therapy, the cost of dual therapy can range between USD 18,000 and 36,000, and the addition of telaprevir increases the cost from USD 48,000 to 85,000 . It has been recently demonstrated that triple therapy is a cost-effective strategy as compared to SOC dual therapy for treating HCV genotype 1 infected patients irrespective of IL28B genotype [13].

The purpose of this study is to introduce an economic model that identifies treatment-naïve individuals infected with HCV genotypes 2 or 3 who are also at risk for relapse or nonresponse to dual therapy (based on the IL28B 
genotype) and to determine when it is cost-effective to treat them with response-guided triple therapy. Specifically, we aim to model both the short- and long-term economic impact of incorporating triple therapy in patients with certain IL28B SNPs compared with SOC (without IL28B genotyping) for treating patients with HCV genotype 2 or 3 infection.

\section{Materials and Methods}

\section{Approach}

The population of interest was treatment-naïve, non-cirrhotic patients with HCV genotype 2 or 3 infection and the outcomes of interest were the 'treatment cost per SVR achieved', and 'healthcare cost per quality-adjusted life-year (QALY) gained'. The subjects assessed by this model are assumed to be of Caucasian origin. Sensitivity analysis was conducted to examine the impact of assumptions on both the short- and long-term models. The costrelated components in this study consist of the direct healthcare costs to the patient and payor. Medication costs (USD) were defined as the average sales price of the drug in 2012. The dosing of pegylated interferon and ribavirin paralleled the dosing under SOC therapy for genotype 1 infected patients. Patients were assumed to have been treated with pegylated interferon at $180 \mu \mathrm{g}$ weekly and ribavirin $400 \mathrm{mg}$ twice daily for 24 weeks. Telaprevir dosing (for the triple therapy threshold analysis) is $750 \mathrm{mg} 3 \times$ daily for 12 weeks, with the induction of this therapy based on host genotype and response to therapy.

\section{Short-Term Model}

The decision tree in figure 1 models the health state pathways (and the corresponding costs and transition probabilities) for the SOC therapy regimens implemented in the studies by Sarrazin et al. [7] and Lindh et al. [14]. For modeling the Sarrazin et al. study [7], the treatment arms were stratified by HCV genotype, while the treatment arms modeling the study by Lindh et al. [14] were stratified by the duration of therapy, which was either 12 or 24 weeks. For clarity, the treatment arms consisting of the HCV genotype 2 $(\mathrm{n}=77)$, HCV genotype $3(\mathrm{n}=190)$, and combined genotype 2 and 3 cohorts $(n=267)$ from the Sarrazin et al. study [7] were designated as Treatment Arms 1, 2 and 3, respectively. The 12-week $(n=175)$ and 24-week $(n=166)$ therapy regimens modeled in the study by Lindh et al. [14] were designated as Treatment Arms 4 and 5, respectively. Treatment Arm 3 was stratified by the sequence variants at rs12979860 and rs8099917, while Treatment Arms 4 and 5 were stratified by the sequence variants at rs 12979860 .

While the Sarrazin et al. study [7] reported outcomes only in patients who had completed SOC therapy with a known virologic outcome, the Lindh et al. study [14] also reported outcomes for patients who had not adhered to the study protocol or who had discontinued therapy early due to adverse events or personal choice.

Two-sample $\mathrm{Z}$ tests were used to test for statistical significance between the baseline characteristics of the 2 studies. The difference in the proportion of males between the 2 studies was significant at a $95 \%$ confidence level, but insignificant at a $99 \%$ confidence level. No significant difference was found between the studies in the ra- tios of HCV genotype 2 to genotype 3 . The mean ages in the Sarrazin et al. [7] and Lindh et al. [14] studies (45.6 and 42 years, respectively) were found to be statistically different at a $95 \%$ confidence level. This study calculated costs and quality-adjusted life expectancies (QALEs) for 2 cohorts: the mean treatment age for the subjects in cohort A was 43.6 years (the weighted average age of the subjects from the 2 studies), while the mean treatment age for the subjects in cohort B was 55 years (representing the typical age of most newly-diagnosed patients with HCV). All analyses were completed using TreeAge Pro 2012 (TreeAge Software, Inc., Williamstown, Mass., USA).

\section{Time Frame}

Treatment costs were accrued over a time horizon ranging from 0 to 74 weeks, reflecting up to 48 weeks of therapy and 26 weeks of post-therapy laboratory tests to assess whether the patients achieve SVR. The time horizon for treatment costs was limited to 74 weeks, as patients that achieve SVR are not administered any additional treatment medications and undergo no further laboratory testing. The health state probabilities and the prevalence of the various IL28B SNPs were gathered by a retrospective review of the literature [15] or from expert consensus when no data were publicly available.

\section{Model Inputs}

The costs of the treatment stages were calculated based on medication and laboratory test expenditures accrued over the duration of the health state in question. For the short-term analysis, this study only considered the explicit costs associated with these treatments; therefore, patient time costs were not included in the calculations.

The expected short-term cost for a particular health state was calculated by multiplying the probability that a patient transitions to that health state (given the patient's current health state in the model) by the treatment cost resulting from transitioning to that particular state. Health states included nonresponse to treatment, RVR, extended rapid virologic response, SVR, and relapse. Nonresponse was defined as HCV RNA levels exceeding 1,000 IU/ml at treatment weeks 4 or 12 [3], RVR as having undetectable HCV RNA levels at week 4, extended rapid virologic response as having undetectable HCV RNA levels at weeks 4 and 12, SVR as having undetectable HCV RNA levels for at least 24 weeks following treatment, and relapse as subjects whose HCV RNA levels became undetectable at some point during therapy, but rose to detectable levels during therapy or up to 6 months after completion of therapy. Each distinct health state was associated with a distinct treatment state. The health state transition probabilities for the various pathways in the decision tree were used to conduct a cohort simulation on the short-term model. These probabilities, along with the source of the data, are listed in online supplementary table 1 (see www.karger.com/doi/10.1159/000365939 for all online suppl. material).

Monte Carlo simulation was used to estimate the costs and effectiveness of the 5 treatment arms. This type of simulation was chosen for its ability to address both second-order (parameter) and first-order uncertainty. First-order uncertainty arises due to variability among individuals and is addressed by running random trials (microsimulation). On the other hand, second-order simulation addresses uncertainty surrounding parameter values by repeating the microsimulation over multiple iterations. Mon- 


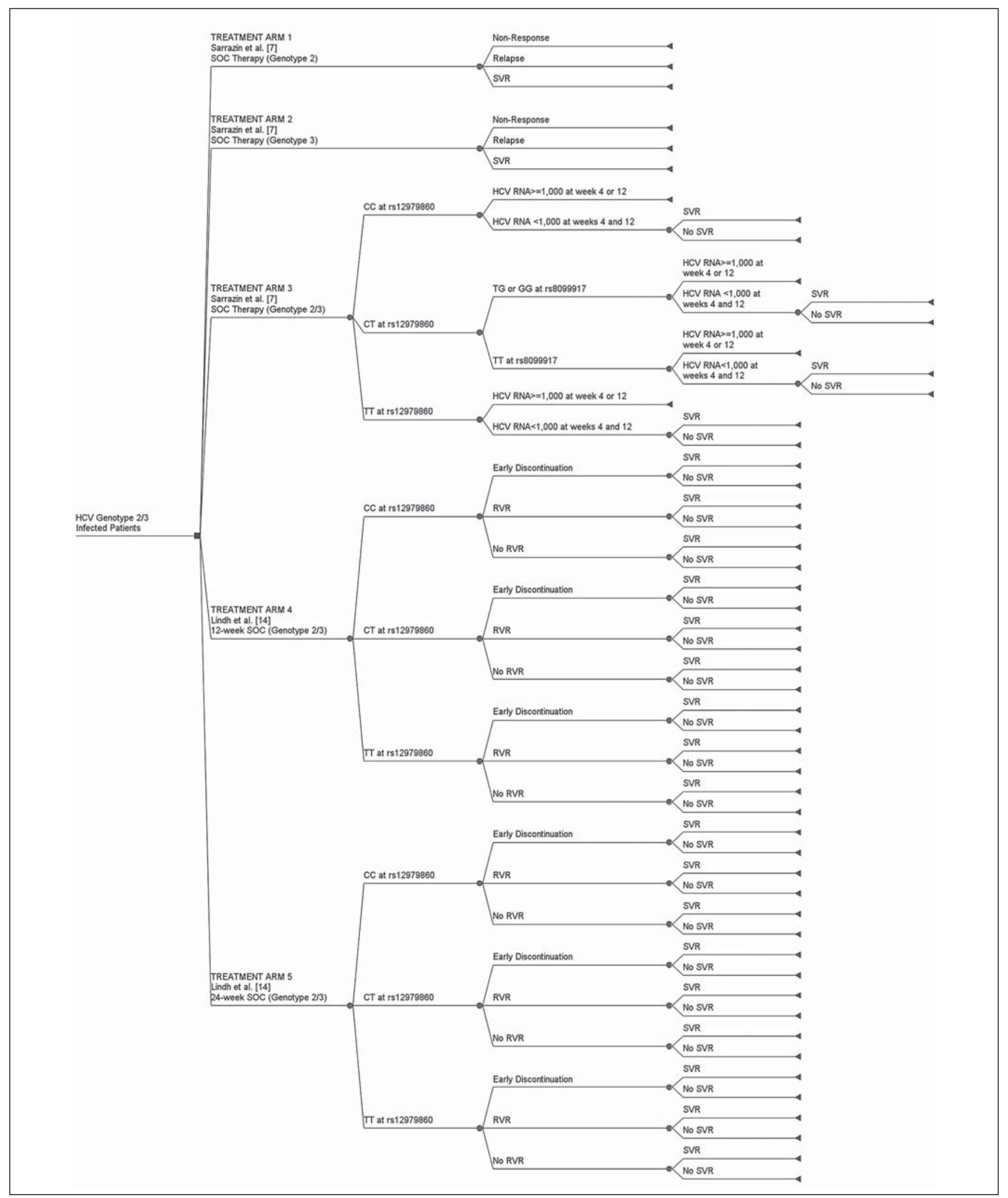

Fig. 1. Decision tree model for short-term costs and outcomes for Treatment Arms 1-5.

Cost-Effectiveness of Treatments for Hepatitis C Genotypes 2 or 3
Public Health Genomics 2014;17:306-319 DOI: $10.1159 / 000365939$ 
te Carlo simulation consisting of 10,000 hypothetical subjects was conducted on each treatment arm in the model. The simulation was repeated for 1,000 iterations, and the treatment costs and SVR rates were averaged to improve accuracy and minimize variability.

\section{Unit Costs}

The average sales price of ribavirin and pegylated interferon and their recommended dosages $[12,16,17]$ for treating patients with $\mathrm{HCV}$ infection were used for calculating costs (online suppl. table 2). Using the listed costs and dosages, the medication cost for the treatment state consisting of 24 weeks of pegylated interferon and ribavirin equates to USD 20,232.24. Furthermore, each encounter resulting in laboratory testing added an additional USD 75.24 in costs to account for the fees associated with conducting these tests $[15,18]$.

\section{Assumptions}

The short-term model makes 4 primary assumptions. Since current SOC guidelines recommend discontinuation of therapy in patients with HCV RNA levels $>1,000 \mathrm{IU} / \mathrm{ml}$ at weeks 4 and 12, we assume that nonresponses are uniformly distributed between the 4 th and 12th weeks of treatment. Therefore, for Treatment Arms 1-3, laboratory test and medications costs for patients that were nonresponsive to therapy were calculated based on 8 weeks of treatment. Second, since relapse occurs after the completion of treatment, we assumed that relapsers bear the expected cost of a complete course of therapy. Similarly, because SVR is achieved only after successful completion of therapy, we assumed that patients who achieve SVR also bear the expected cost of a full therapy regimen. For Treatment Arms 1-3, patients who attained SVR or who had relapsed underwent 24 weeks of SOC therapy. Third, based on the previous literature [7] showing that the vast majority of the subjects were non-cirrhotic, we also assumed that the transition probabilities utilized in our model reflect a population that is treatment-naïve and non-cirrhotic [7]. Lastly, the SVR rates for Treatment Arms 4 and 5 accounted for patients that were nonadherent to the treatment protocol, and we assumed that these patients also bear the expected cost of a full therapy regimen.

\section{Long-Term Model}

The long-term analysis was run on the subjects following each of the treatment arms from figure 1. The long-term analysis was conducted through the use of Markov modeling, using SVR, no SVR, compensated cirrhosis, decompensated cirrhosis (hepatic encephalopathy, variceal bleed and ascites), HCC, liver transplant, and death from liver disease as the health states of interest (fig. 2). The long-term model assessed the health-related quality-of-life (HRQoL) impact of the aforementioned health states of interest and the healthcare costs for the treatment and care associated with these conditions.

\section{Time Frame}

Long-term costs and health outcomes were analyzed over the time interval ranging from the end of the short-term model (i.e. the therapy phase) to the death of the patient (all-cause mortality). The cycle length in the Markov model was defined as 12 months, and a half-cycle correction was added to each health state.

\section{Model Inputs}

The SVR rates from the short-term model determined the initial distribution of subjects among the 'SVR' and 'no SVR' Markov states. Since the 5 treatment arms resulted in 5 distinct SVR rates, the long-term analysis was conducted separately for each of the treatment arms. Health state costs for the various conditions were gathered from the medical literature, and the HRQoL weights and transition probabilities among these health conditions were gathered from published trial studies $[15,19]$ (online suppl. table 3 ). The appropriate health state costs, quality-of-life weights, and health state transition probabilities used in our Markov model were gathered by identifying matching health states in previously published trials. The trials our model was based on are the following: (1) they were conducted on HCV genotype 2 or 3 infected patients, (2) they identified the annual transition probabilities, costs, and HRQoL weights for the health states, and (3) they were conducted in a manner that minimized the potential for error and bias. The transition probabilities, costs, and HRQoL weights for the various Markov health states are listed in online supplementary tables 1, 2 and 3, respectively.

Costs and QALEs were calculated from the model; both costs and HRQoL were discounted at 3\% per annum. Recent studies have shown that $1.75 \%$ of non-cirrhotic HCV infected patients that achieve SVR develop HCC after 5 years post-therapy [20]. Assuming that transitions occur uniformly within the 5-year time frame, the annual probability of transitioning from the SVR state to the HCC state was calculated as $0.35 \%$.

The first-year decompensated cirrhosis health state in the Markov model is composed of the combination of diuretic sensitive ascites (62\%), variceal hemorrhage (28\%) and hepatic encephalopathy (10\%) [21]. The transition probability of first-year decompensated cirrhosis to death was estimated by multiplying the proportions of ascites, variceal hemorrhage, and hepatic encephalopathy in patients with first-year decompensated cirrhosis by their relative mortality rates and then summating the results. The transition probability of subsequent-year decompensated cirrhosis to death was estimated in the same manner but with ascites, variceal hemorrhage, and hepatic encephalopathy comprising 73.4, 22.3, and $4.3 \%$ of patients with subsequent-year decompensated cirrhosis, respectively [21]. The costs and HRQoL weights for first and subsequent-year decompensated cirrhosis were calculated by the sum of the proportions of ascites, variceal hemorrhage, and hepatic encephalopathy multiplied by their respective costs or HRQoL weights.

Modeling All-Cause and Other-Cause Mortality over Time

The probability of 'other-cause mortality', which was defined as the probability of death occurring from any cause other than $\mathrm{HCV}$ or HCV-related comorbidities in the current cycle, was added to the Markov model. This allowed the model to generate realistic life expectancies for individuals who did not succumb to liver-related death. The mean age at any given cycle during the Markov simulation was calculated by the sum of the average age of the cohorts during therapy (i.e. 43.6 and 55 years) and the current stage in the simulation. The probability of all-cause mortality at a certain age was obtained from the National Center for Health Statistics' 2008 life tables [22]. The life tables provided the annual probability of death at each age between birth and 100 years. 


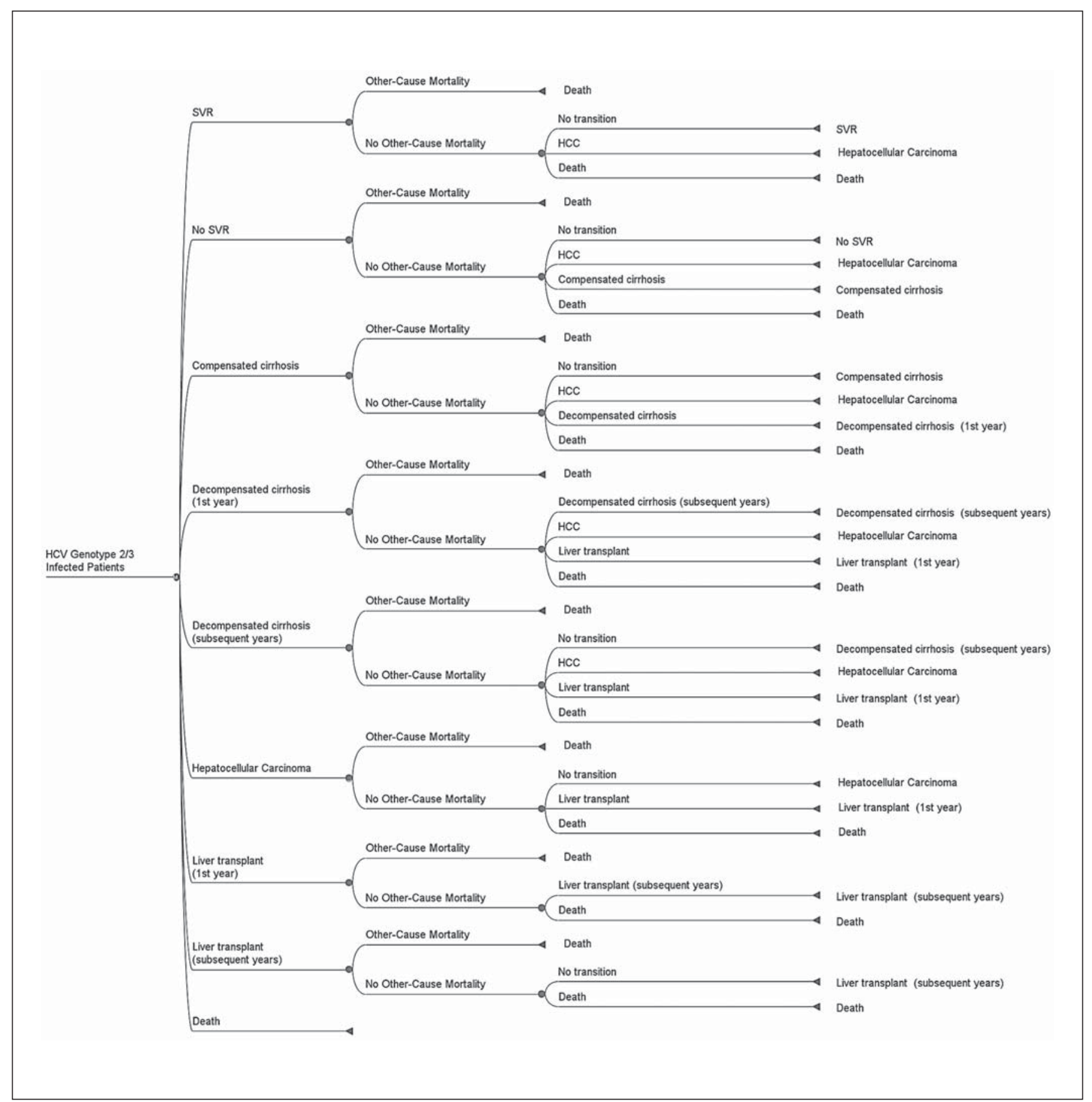

Fig. 2. Markov model for long-term costs and outcomes.

The probability of HCV-related death among the general population was calculated as the product of the $\mathrm{HCV}$ prevalence rate (for ages 40-54) and the probability of HCV-related death given the current health state. To avoid double-counting HCV-related deaths, the probability of HCV-related death among the general population was subtracted from the probability of all-cause mortality. Cohort subjects that did not transition to other-cause mortality would either remain in the current health state, transition to another health state, or transition to death from liver-related disease. 
Assumptions

It is widely accepted that the achievement of SVR is associated with decreased mortality and a decreased risk of developing liverrelated complications. Thus, the long-term model assumes that SVR rates achieved in the short-term model are the main factor influencing long-term costs and QALE. This assumption is reflected in the long-term model's use of the SVR rate as its primary input.

\section{Triple Therapy Threshold Analysis}

Costs and outcomes were modeled for cohorts of patients with HCV genotypes 2 or 3 with either universal triple therapy or triple therapy restricted based on $I L 28 B$ genotype. In the cohorts where triple therapy was restricted to certain $I L 28 B$ genotypes, individuals at high risk for relapse would be given response-guided adjunctive therapy with telaprevir in an effort to increase SVR. Short- and long-term costs were calculated for treatment-related costs as well as the consequences of nonresponse and complications of longterm HCV infection.

The decision trees depicted in figures 3, 4 were adapted from guidelines for the treatment of HCV genotype 1 with adjunctive protease inhibitor therapy and recommendations based on the ADVANCE and SPRINT-2 trials [8,9], illustrating the use of IL $28 B$ genotyping to guide triple therapy in HCV genotype 2 or 3 infected patients.

The decision tree in figure 3 was designed to model the therapy regimen, $I L 28 B$ SNPs of interest, the distribution of the IL28B gene variants among the cohort, and outcomes observed in HCV genotype 2 or 3 infected patients in the Sarrazin et al. study [7]. Similarly, the decision trees in figures $4 \mathrm{a}$, b model the 12 and 24-week therapy regimens, the $I L 28 B$ SNP of interest, the distribution of $I L 28 B$ gene variants among the study subjects, and the outcomes observed in the study by Lindh et al. [14]. Figures 3, 4 depict the IL28B genotype-guided treatment algorithms that were developed to risk stratify patients with a concern for nonresponse to $\mathrm{HCV}$ therapy and target them for intensive treatment. This protocol predicts which patients would have a less favorable response to standard treatment, and then this would qualify them for additional therapy with a protease inhibitor.

\section{Model Inputs}

Using the predicted cost of a 12-week regimen of telaprevir, triple therapy treatment costs were calculated for the various treatment arms. The threshold (i.e. minimum SVR rate) at which triple therapy becomes a cost-effective alternative to SOC was calculated for each of the treatment arms. The triple therapy arm was compared to each of the SOC Treatment Arms 1-5 to determine, in each case, the SVR rate and the treatment cost needed to reach the cost-effectiveness threshold. This study used a USD 50,000 per QALY threshold in the analysis, which is a commonly used threshold standard to denote cost-effectiveness in medical interventions. For example, a review by Neumann et al. [23] of 228 pharmacoeconomic studies revealed that USD 50,000 per QALY gained was the most commonly used threshold standard.

Lindh et al. [14] recognized that a patient's sequence variant at rs12979860 was highly predictive of the probability of SVR and the first phase of viral elimination. Since the sequence variant at rs8099917 was not examined in the Lindh et al. study [14], the addition of telaprevir in Treatment Arms 4 and 5 was based on only the rs12979860 SNP. Treatment Arms 4 and 5 were each separated into 2 subdivisions. For Treatment Arms 4a and 5a, only subjects with the TT SNP at rs12979860 were given triple therapy (fig. 4a); for Treatment Arms 4b and 5b, subjects with the CT or TT SNPs at rs12979860 were given triple therapy (fig. 4b). The cost-effectiveness thresholds were calculated for cohort A and B.

\section{Unit Costs}

The average sales price of telaprevir and its recommended dosage $[12,16,17]$ for treating patients with HCV infection is listed in online supplementary table 2 . The telaprevir regimen was derived from HCV genotype 1 dosing. The medication cost for the treatment state consisting of 24 weeks of pegylated interferon and ribavirin and 12 weeks of telaprevir is USD 26,323.92. The triple therapy treatment arm includes a fixed cost of USD 890.00 to account for the cost of $I L 28 B$ genotyping, consisting of the approved laboratory fee and the Medicare reimbursement cost of IL28B genotyping at our facility. Lastly, USD 75.24 in costs were added to encounters resulting in laboratory testing to account for these fees $[15,18]$.

\section{Assumptions}

Both decision trees assume that differences in the genotypes of the $I L 28 B$ gene are reflected by differences in the viral loads at 4 and 12 weeks, which ultimately influence the probability that a patient achieves extended rapid virologic response and subsequently, SVR, given a specific 2 -sequence variant in IL28B. The decision trees in figure $4 \mathrm{a}, \mathrm{b}$ assume that early discontinuation of therapy occurs, on average, halfway through the therapy regimens, and that these patients bear half the expected cost of a full therapy regimen. The analysis assumed that nonresponse rates matched those observed under SOC. The analysis further assumed that treatment was discontinued in patients found to be nonresponsive to triple therapy and, therefore, did not accrue any additional medication or laboratory test costs.

\section{Sensitivity Analysis}

The nonresponse rates used to model the addition of telaprevir to SOC therapy in patients following Treatment Arms 1, 2 and 3 were varied in the sensitivity analysis to account for uncertainty surrounding response to telaprevir in this cohort of subjects. Furthermore, the early discontinuation and RVR transition probabilities for the various $I L 28 B$ sequence variants in the figure 4 decision trees were derived from the observed early discontinuation rates and RVR rates for the corresponding sequence variants observed in the Lindh et al. study [14]. These probabilities were used as baseline values to predict the treatment costs of 12 - and 24-week IL28B genotype-guided therapy regimens if outcomes were not affected by the addition of telaprevir. These early discontinuation and RVR transition probabilities were varied during sensitivity analysis to reflect changes in health outcomes due to the addition of telaprevir. The variables in the short-term model most cost-sensitive to uncertainty (based on contribution to variance) are visually represented as a tornado diagram in online supplementary figure 1. Online supplementary table 4 provides the sensitivity analysis results for all variables in the short-term model, regardless of the variables' levels of sensitivity to uncertainty.

Sensitivity analysis was also conducted for the transition probabilities used in the long-term model. Lower and upper limits for the various parameters were used to the extent to which they were available in the medical literature and were valid reflections of the parameter of interest. Appropriate adjustments were made when 


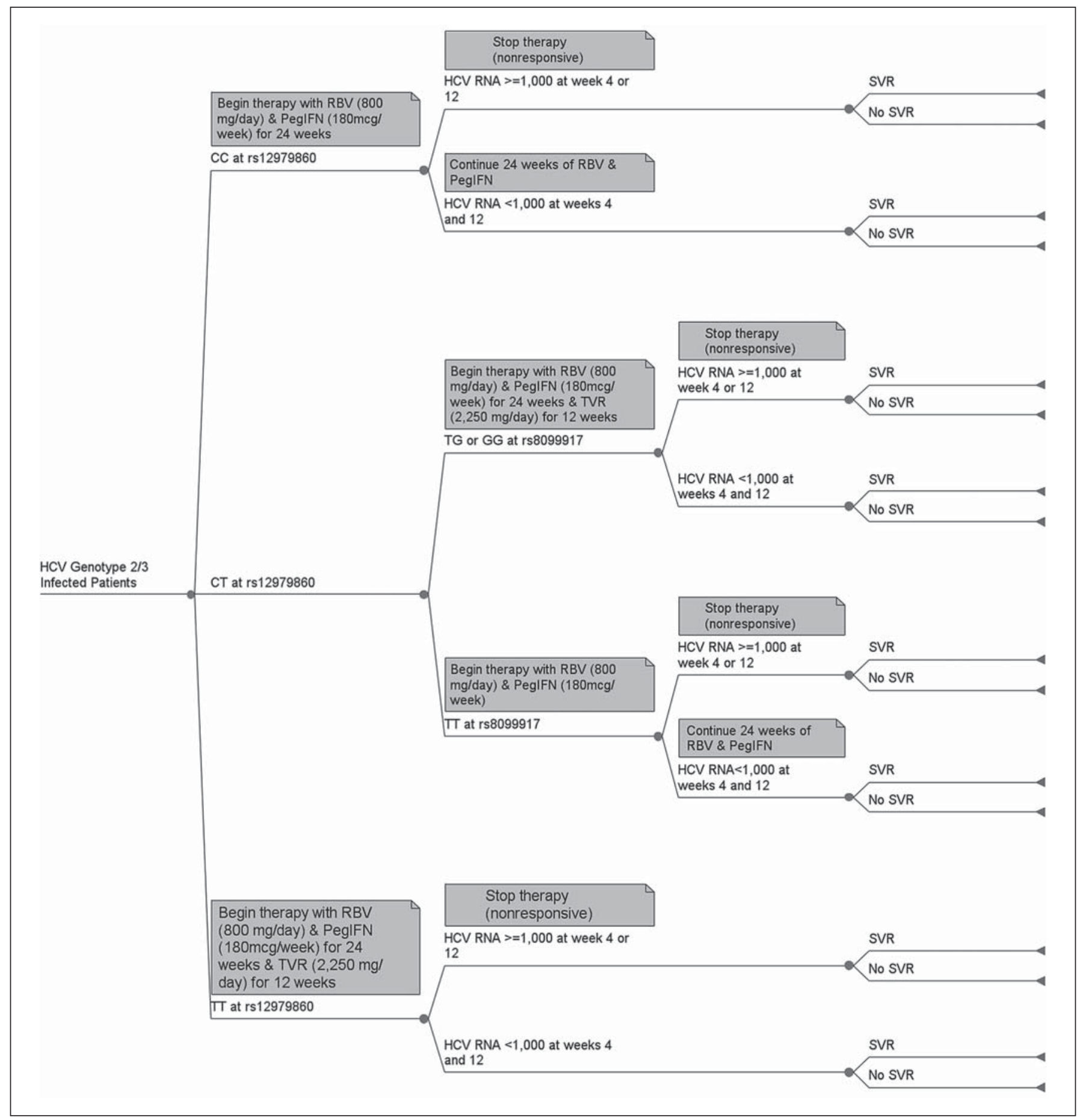

Fig. 3. Short-term model depicting the use of $I L 28 B$ genotyping and telaprevir for patients in the Sarrazin et al. study [7].

the tested variable was correlated with one or more other parameters. The upper and lower limits for the various parameters are listed in online supplementary tables 1 and 3. The long-term sensitivity analysis was conducted for each of the treatment arms for both cohorts A and B. The tornado diagram in online supplemen-

Cost-Effectiveness of Treatments for Hepatitis C Genotypes 2 or 3 tary figure 2 depicts the results of the sensitivity analysis for the most cost-sensitive long-term variables, while the tornado diagram in online supplementary figure 3 includes those variables most sensitive to variations in the HRQoL weights. The tornado diagrams specify the age cohort and the contribution to variance 


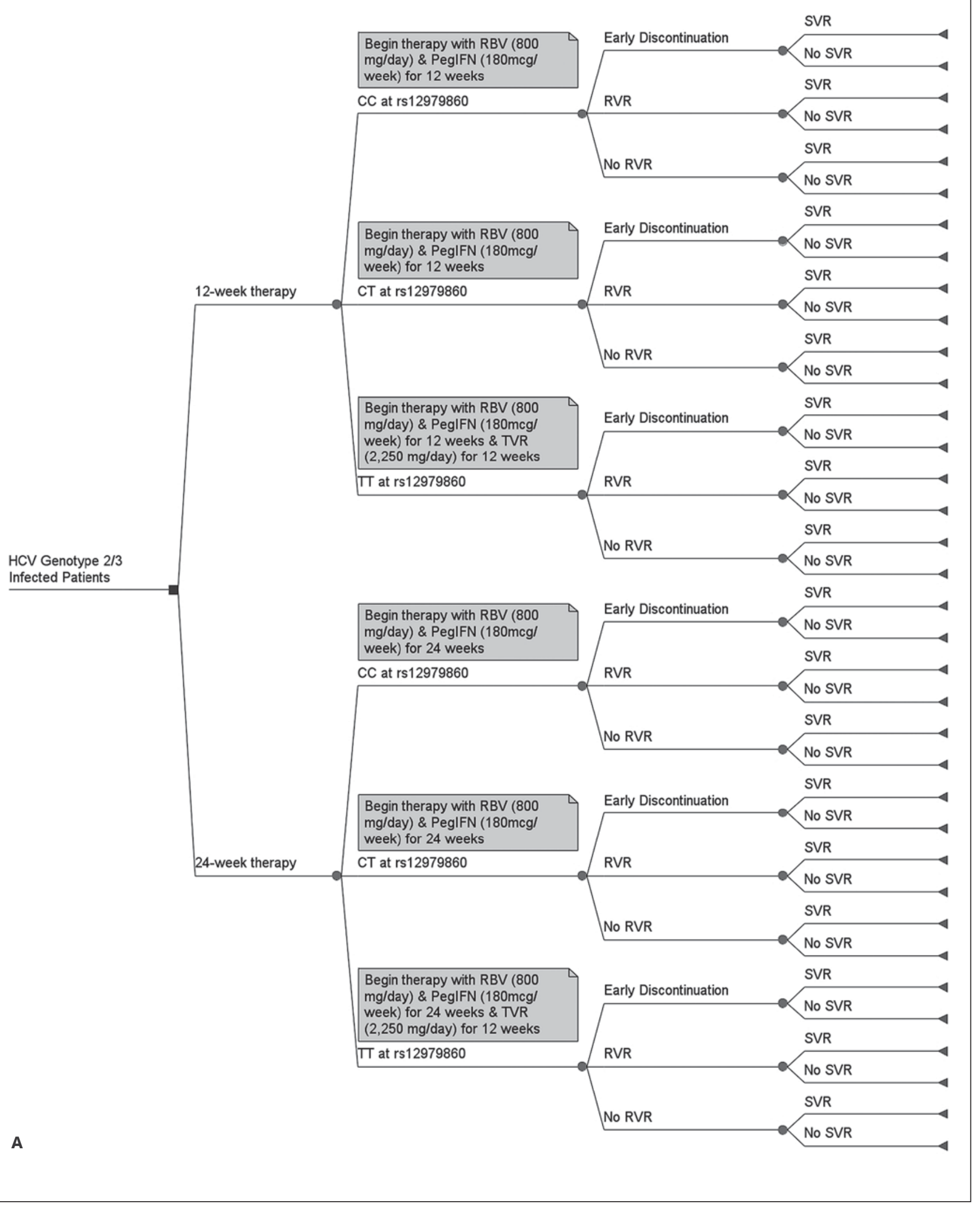

4

(For legend see next page.)

Bock/Fairley/Smith/Maeng/Pitcavage/ Inverso/Williams 


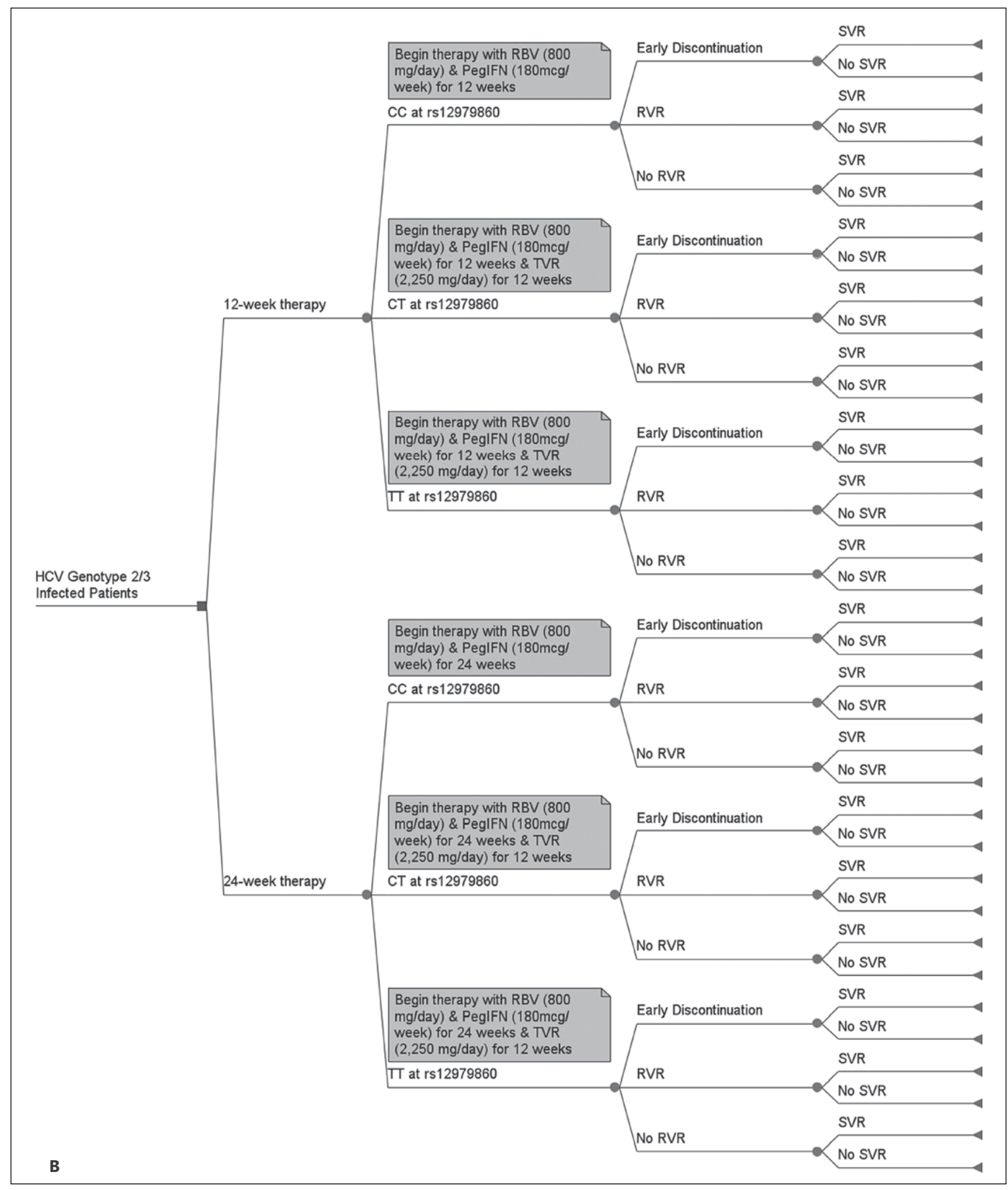

Fig. 4. Decision tree depicting the use of IL28B genotyping and telaprevir for patients in the Lindh et al. study [14]. A TT at rs12979860 only. B TT and CT at rs12979860.

Cost-Effectiveness of Treatments for Hepatitis C Genotypes 2 or 3
Public Health Genomics 2014;17:306-319 DOI: 10.1159/000365939 


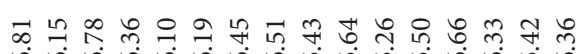

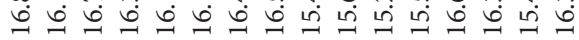

\&

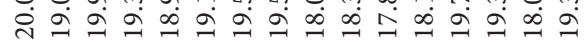
กี

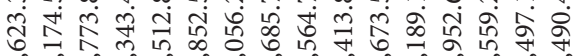

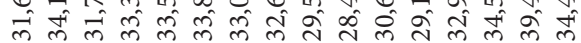

for each variable tested. Online supplementary tables $5 \mathrm{a}, \mathrm{b}$ and $6 \mathrm{a}$, $\mathrm{b}$ provide the sensitivity analysis results for cohorts $\mathrm{A}$ and $\mathrm{B}$, respectively.

\section{Results}

\section{SOC Short- and Long-Term Costs and Outcomes}

The short-term costs and outcomes for each of the treatment arms and the IL28B sequence variants (when specified) are summarized in table 1 . The results summarized in table 1 are based on a cohort (A) with a mean treatment age of 43.6 years and a cohort (B) with a mean treatment age of 55 years. Since the probability of achieving SVR is not influenced by age [24], the short-term costs and SVR rates are identical in the 2 cohorts. The HRQoL weights listed in online supplementary table 3 [15] and the SVR rates resulting from the various treatment arms were incorporated into the Markov model, and the resulting total costs and QALEs are shown in table 1.

\section{Triple Therapy Threshold Analysis Results}

The predicted triple therapy treatment costs, cost-effectiveness thresholds, and the relative percent changes in observed SVR rates needed to reach the cost-effectiveness threshold are presented in table 2 , representing cohorts A and $B$. When the administration of triple therapy is guided by IL28B genotype, relatively small increases in SVR rates are needed to reach a cost-effectiveness threshold.

In cohort $\mathrm{A}$, for the combined HCV genotype 2 and 3 cohort in Treatment Arm 3, the administration of triple therapy to patients having the TG/GG variant at rs 8099917 or the TT variant at rs 12979860 only requires a $2.63 \%$ improvement (relative to SOC) in the SVR rate to reach a USD 50,000 per QALY cost-effectiveness threshold. Administering triple therapy to patients with the TT variant at rs12979860 only requires a 1.66 and a 1.4\% improvement in SVR for the HCV genotype 2 and 3 cohorts in Treatment Arm 4 and Treatment Arm 5, respectively. On the other hand, the administration of triple therapy to all patients, regardless of $I L 28 B$ variant, would require a $7.91 \%$ improvement in SVR for the HCV genotype 2 cohort (Treatment Arm 1) and a 9.06\% improvement for the HCV genotype 3 cohort (Treatment Arm 2) in order to reach a USD 50,000 per QALY threshold.

In cohort $\mathrm{B}$, administering triple therapy to subjects in Treatment Arm 3 who have the TG/GG variant at rs 8099917 or the TT variant at rs 12979860 only requires a $3.72 \%$ improvement (relative to SOC) in the SVR rate to reach the USD 50,000 per QALY threshold. For Treat- 
Table 2. Outcomes for the threshold analysis for cohort A and B

\begin{tabular}{|c|c|c|c|c|c|c|c|c|}
\hline \multirow[t]{2}{*}{ Ref. } & \multirow{2}{*}{$\begin{array}{l}\text { SOC } \\
\text { duration, } \\
\text { weeks }\end{array}$} & \multirow[t]{2}{*}{ HCV cohort } & \multirow[t]{2}{*}{ Telaprevir recipients } & \multirow[t]{2}{*}{$\begin{array}{l}\text { Therapy cost, } \\
\text { USD }\end{array}$} & \multicolumn{2}{|c|}{$\begin{array}{l}\text { Cost-effectiveness threshold, } \\
\text { SVR rate }\end{array}$} & \multicolumn{2}{|c|}{ Threshold increase from SOC } \\
\hline & & & & & cohort A & cohort B & cohort A & cohort B \\
\hline [7] & 24 & Treatment Arm 1 & All SNPs & $46,294.49$ & $\geq 94.85$ & $\geq 97.67$ & 7.91 & 11.11 \\
\hline [7] & 24 & Treatment Arm 2 & All SNPs & $44,334.71$ & $\geq 80.92$ & $\geq 83.70$ & 9.06 & 12.80 \\
\hline [7] & 24 & Treatment Arm 3 & $\begin{array}{l}\text { TG/GG (rs8099917) or } \\
\text { TT (rs12979860) }\end{array}$ & $27,613.03$ & $\geq 83.85$ & $\geq 84.74$ & 2.63 & 3.72 \\
\hline [14] & 12 & Treatment Arm 4b & CT or TT (rs12979860) & $24,881.22$ & $\geq 64.64$ & $\geq 66.11$ & 6.49 & 8.91 \\
\hline [14] & 24 & Treatment Arm 5b & CT or TT (rs12979860) & $37,056.93$ & $\geq 82.82$ & $\geq 84.74$ & 5.50 & 7.95 \\
\hline
\end{tabular}

The average cost of USD 27,613.03 in the Sarrazin et al. [7] HCV genotype 2/3 cohort (Treatment Arm 3) was based on USD 21,181.43 and 43,647.88 in treatment costs for the CC and TT variants at rs12979860, respectively, and USD 45,100.82 and 20,576.36 in treatment costs for the TG/GG and TT variants at rs8099917, respectively. Therapy costs include the cost of pegylated interferon, ribavirin, laboratory tests and/or protease inhibitors. All cohort data are percentages.

ment Arms 4 and 5, administering triple therapy to patients with the TT variant at rs12979860 only requires a 2.17 and a $2.11 \%$ improvement in SVR, respectively. The administration of triple therapy to all patients, irrespective of $I L 28 B$ variant, requires an $11.11 \%$ improvement in SVR for the HCV genotype 2 cohort (Treatment Arm 1) and a $12.80 \%$ improvement for the genotype 3 cohort (Treatment Arm 2) in order to reach a USD 50,000 per QALY threshold.

\section{Discussion}

Therapy for HCV infection can result in serious complications and generate significant healthcare costs. We applied a method to identify those patients likely to benefit from costly adjunctive therapeutic regimens.

IL28B genotyping has demonstrated the importance of 2 SNPs (rs12979860 and rs8099917) that are strongly correlated with the probability of obtaining a favorable response to treatment. Sarrazin et al. [7] reported that of genotype 2 or 3 infected patients obtaining an SVR, 87.4\% had the CC genotype at rs12979860. 19.2 versus 3.4\% of patients homozygous for the recessive (TT) and dominant alleles (CC), respectively, had virologic nonresponse [7]. Foster et al. [12] further demonstrated statistically significant differences in SVR rates with combined genotypes when the SNP rs12979860 was heterozygous CT. In this circumstance, combining the second SNP, rs8099917, resulted in significantly different probabilities of patients obtaining an SVR. In these HCV genotype 1 infected

Cost-Effectiveness of Treatments for

Hepatitis C Genotypes 2 or 3 patients, SVR rates of 38 versus $21 \%$ were observed for the combined genotypes rs12979860CT/rs8099917TT and $\mathrm{rs} 12979860 \mathrm{CT} / \mathrm{rs} 8099917 \mathrm{TG}$, respectively; therefore, the use of both SNPs (rs12979860 and rs8099917) was incorporated into the short-term model. Patients with unfavorable chances of obtaining SVR based on SNPs rs12979860TT, rs12979860CT/rs8099917TG or rs12979860CT/rs8099917GG received intensive treatment with triple therapy as the initial therapeutic regimen.

The potential limitations of this study include population bias and limited data for certain transition probabilities. The modeling and results above are based on a pool of patients that are primarily Caucasian and of European descent and, therefore, reflect an IL28B distribution for this population. A different distribution of $I L 28 B$ frequencies may produce differing results. This parameter is modeled on prevalence data from currently available literature [7]. Furthermore, some of the transition probabilities in certain treatment pathways were based on expert consensus given the paucity of literature regarding response rates to triple therapy in genotype 2 and 3 infected patients. However, recent studies documenting IL28B genotype frequencies and observed transition probabilities in response to dual therapy in HCV genotypes 2 and 3 infected patients have mitigated the impact of unknowns. Sensitivity analysis confirmed the modest impact of this uncertainty at a $95 \%$ confidence level.

We externally validated our model against 2 studies that analyzed the cost-effectiveness of treatment using pegylated interferon/interferon and/or ribavirin. The 
2003 study by Seibert et al. [15] evaluated the cost-effectiveness of various drug-based treatments for patients with HCV genotype 2 or 3 infection. The 2004 study by Sullivan et al. [25] evaluated the incremental cost-effectiveness of pegylated interferon $\alpha$-2a plus ribavirin versus interferon $\alpha$-2a plus ribavirin in HCV genotype 1 and HCV non-genotype 1 infected patients.

In the Seibert et al. study [15], SVR rates of 47, 54 and $61 \%$ resulted in discounted QALEs of 16.8, 17.0 and 17.3 years, respectively. After incorporating the aforementioned SVR rates, the HRQoL weights, and the health state transition probabilities used in the Seibert et al. study [15], our model predicted discounted QALEs of $16.92,17.34$ and 17.75 years for SVR rates of 47,54 and $61 \%$, respectively. The QALEs predicted by our model compare well to the Seibert et al. study's predicted QALEs [15], suggesting that our model serves as a valid estimator of QALE among patients with HCV genotype 2 or 3 infection.

After incorporating the health state transition probabilities, HRQoL weights, and SVR rates used in the 2004 Sullivan et al. [25] study into our model, it estimated a 0.74 year increase in QALE in HCV genotype 1 patients and a 1.11 year QALE increase in HCV non-genotype 1 patients. These results compare well to the study by Sullivan et al. [25], which estimated 0.70 and 1.05 year increases in QALE among HCV genotype 1 and HCV nongenotype 1 patients, respectively.

The total costs predicted by our model moderately differed to the costs predicted in the Seibert et al. [15] study. However, these differences are largely due to differences in variables. For example, our study includes the possibility of patients who achieve SVR may develop HCC later in time - an assumption that increases the predicted longterm costs by USD 3,546. Furthermore, as the study [15] mentions, the probability of a liver transplant for patients with decompensated cirrhosis is $0.9 \%$ higher in the US than in the German cohort analyzed in the study [15]. The higher probability of liver transplantation increases predicted long-term costs by an additional USD 2,493. Finally, the probability of liver transplantation among patients with HCC was included in our model, adding roughly USD 3,970 in liver transplantation costs due to HCC. After accounting for these differences, our model's predicted costs and the Seibert et al. study's [15] predicted costs differ by less than $3.8 \%$.

Studies have demonstrated that triple therapy represents a cost-effective strategy for HCV genotype 1 infected patients. Despite the fact that HCV genotype 2 or 3 infected individuals generally achieve higher SVR rates with SOC therapy, there remains an opportunity to identify patients who may have an unsatisfactory response to SOC therapy. For these patients, pretreatment use of IL28B profiling may augment response rates by identifying individuals that harbor unfavorable polymorphisms and, therefore, direct them to more intensive treatment regimens that improve SVR at an acceptable cost-effectiveness threshold. This Markov model illustrates the need to consider a personalized approach to optimize medical therapy by assessing short- and long-term economic and personal health outcomes in chronic illnesses.

\section{Acknowledgements}

We would like to acknowledge and extend our gratitude to Dr. Scott Grosse, research economist at the Centers for Disease Control and Prevention, for his assistance in helping develop the concept for this paper and to Dean Parry, director of the clinical pharmacy program at Geisinger Health System, for his assistance in gathering the cost information of the various pharmaceuticals analyzed in this study.

Dr. Williams and Mr. Bock received funding support from NIH 1 U01 HG006382-02.

\section{References}

-1 Smith BD, Morgan RL, Beckett GA, Falck-Ytter Y, Holtzman D, Ward JW: Hepatitis C virus testing of persons born during 1945 to 1965: recommendations from the Centers for Disease Control and Prevention. Ann Intern Med 2012;157:817-822.

$\checkmark 2$ McCombs JS, Yuan Y, Shin J, Saab S: Economic burden associated with patients diagnosed with hepatitis C. Clin Ther 2011;33: 1268-1280.

$>3$ Ghany MG, Nelson DR, Strader DB, Thomas DL, Seeff LB; American Association for Study of Liver Diseases: An update on treatment of genotype 1 chronic hepatitis $\mathrm{C}$ virus infection: 2011 practice guideline by the American Association for the Study of Liver Diseases. Hepatology 2011;54:1433-1444.

$>4$ Manns MP, McHutchison JG, Gordon SC, Rustgi VK, Shiffman M, Reindollar R, Goodman ZD, Koury K, Ling M, Albrecht JK: Peginterferon alfa-2b plus ribavirin compared with interferon alfa-2b plus ribavirin for initial treatment of chronic hepatitis C: a randomised trial. Lancet 2001;358:958-965.

5 Andriulli A, Mangia A, Iacobellis A, Ippolito A, Leandro G, Zeuzem S: Meta-analysis: the outcome of anti-viral therapy in HCV genotype 2 and genotype 3 infected patients with chronic hepatitis. Aliment Pharmacol Ther 2008;28:397-404.

-6 Fischer J, Böhm S, Scholz M, Müller T, Witt H, George J, Sarrazin C, Susser S, Schott E, Suppiah V, Booth DR, Stewart GJ, van Bömmel F, Brodzinski A, Fülöp B, Migaud P, Berg $\mathrm{T}$ : Combined effects of different interleukin$28 \mathrm{~B}$ gene variants on the outcome of dual combination therapy in chronic hepatitis $\mathrm{C}$ virus type 1 infection. Hepatology 2012;55: $1700-1710$ 
7 Sarrazin C, Susser S, Doehring A, Lange CM, Müller T, Schlecker C, Herrmann E, Lötsch J, Berg T: Importance of IL28B gene polymorphisms in hepatitis $C$ virus genotype 2 and 3 infected patients. J Hepatol 2011;54:415-421.

8 Jacobson IM, McHutchison JG, Dusheiko G, Di Bisceglie AM, Reddy KR, Bzowej NH, Marcellin P, Muir AJ, Ferenci P, Flisiak R, George J, Rizzetto M, Shouval D, Sola R, Terg RA, Yoshida EM, Adda N, Bengtsson L, Sankoh AJ, Kieffer TL, George S, Kauffman RS, Zeuzem S; ADVANCE Study Team: Telaprevir for previously untreated chronic hepatitis C virus infection. N Engl J Med 2011;364: 2405-2416.

-9 Poordad F, McCone J Jr, Bacon BR, Bruno S, Manns MP, Sulkowski MS, Jacobson IM, Reddy KR, Goodman ZD, Boparai N, DiNubile MJ, Sniukiene V, Brass CA, Albrecht JK, Bronowicki JP; SPRINT-2 Investigators: Boceprevir for untreated chronic HCV genotype 1 infection. N Engl J Med 2011;364:11951206.

10 Chayama K, Hayes CN, Abe H, Miki D, Ochi $\mathrm{H}$, Karino Y, Toyota J, Nakamura Y, Kamatani N, Sezaki H, Kobayashi M, Akuta N, Suzuki F, Kumada H: IL28B but not ITPA polymorphism is predictive of response to pegylated interferon, ribavirin, and telaprevir triple therapy in patients with genotype 1 hepatitis C. J Infect Dis 2011;204:84-93.

11 Lin C, Hanzelka BL, Müh U, Kovari L, Bartels DJ, Tigges AM, Miller J, Rao BG, Kwong AD: Telaprevir (VX-950) is a potent inhibitor of HCV NS3-4A proteases derived from genotype non-1 HCV infected patients. J Hepatol 2007;46(suppl 1):S8.
2 Foster GR, Hézode C, Bronowicki JP, Carosi G, Weiland O, Verlinden L, van Heeswijk R, van Baelen B, Picchio G, Beumont M: Telaprevir alone or with peginterferon and ribavirin reduces HCV RNA in patients with chronic genotype 2 but not genotype 3 infections. Gastroenterology 2011;141:881-889.e1.

13 Cammà C, Petta S, Enea M, Bruno R, Bronte F, Capursi V, Cicchetti A, Colombo GL, Di Marco V, Gasbarrini A, Craxi A; WEF Study Group: Cost-effectiveness of boceprevir or telaprevir for untreated patients with genotype 1 chronic hepatitis C. Hepatology 2012; 56:850-860.

14 Lindh M, Lagging M, Färkkilä M, Langeland N, Mørch K, Nilsson S, Norkrans G, Pedersen C, Buhl MR, Westin J, Hellstrand K: Interleukin 28B gene variation at rs12979860 determines early viral kinetics during treatment in patients carrying genotypes 2 or 3 of hepatitis C virus. J Infect Dis 2011;203:1748-1752.

15 Siebert U, Sroczynski G, Rossol S, Wasem J, Ravens-Sieberer U, Kurth BM, Manns MP, McHutchison JG, Wong JB; German Hepatitis C Model (GEHMO) Group; International Hepatitis Interventional Therapy (IHIT) Group: Cost effectiveness of peginterferon alpha- $2 \mathrm{~b}$ plus ribavirin versus interferon alpha$2 \mathrm{~b}$ plus ribavirin for initial treatment of chronic hepatitis C. Gut 2003;52:425-432.

16 Cardinal Health. Pharmaceutical Distribution; Entelligence Version 6.0.

17 United States Department of Veterans Affairs: Hepatitis C, Antiviral TX Strategy, Genotype 2-3. 2006.

18 Centers for Medicare and Medicaid Services, American Medical Association: Clinical Diagnostic Laboratory Fee Schedule 2012.

-19 Llovet JM, Mas X, Aponte JJ, Fuster J, Navasa M, Christensen E, Rodés J, Bruix J: Cost effectiveness of adjuvant therapy for hepatocellular carcinoma during the waiting list for liver transplantation. Gut 2002;50:123-128.
20 Ogawa E, Furusyo N, Kajiwara E, Takahashi K, Nomura H, Maruyama T, Tanabe Y, Satoh T, Nakamuta M, Kotoh K, Azuma K, Dohmen K, Shimoda S, Hayashi J; Kyushu University Liver Disease Study (KULDS) Group: Efficacy of pegylated interferon alpha-2b and ribavirin treatment on the risk of hepatocellular carcinoma of patients with chronic hepatitis C: a prospective, multicenter study. J Hepatol 2013;58:495-501.

21 Salomon JA, Weinstein MC, Hammitt JK, Goldie SJ: Cost-effectiveness of treatment for chronic hepatitis $\mathrm{C}$ infection in an evolving patient population. JAMA 2003;290:228-237.

22 Arias E: United States Life Tables 2008. NVSS 2012;61.

23 Neumann PJ, Greenberg D, Olchanski NV, Stone PW, Rosen AB: Growth and quality of the cost-utility literature, 1976-2001. Value Health 2005;8:3-9.

24 Frei P, Leucht AK, Held U, Kofmehl R, Manser CN, Schmitt J, Mertens J, Rau M, Baur K, Gerlach T, Negro F, Heim M, Moradpour D, Cerny A, Dufour JF, Müllhaupt B, Geier A; Swiss Hepatitis C Cohort Study Group: Elderly age is not a negative predictive factor for virological response to therapy with pegylated interferon-alpha and ribavirin in chronic hepatitis C virus patients. Liver Int 2014;34: 551-557.

25 Sullivan SD, Jensen DM, Bernstein DE, Hassanein TI, Foster GR, Lee SS, Cheinquer $\mathrm{H}$, Craxi A, Cooksley G, Klaskala W, Pettit K, Patel KK, Green J: Cost-effectiveness of combination peginterferon alpha-2a and ribavirin compared with interferon alpha- $2 \mathrm{~b}$ and ribavirin in patients with chronic hepatitis C. Am J Gastroenterol 2004;99:1490-1496. 